\title{
5 Research Square \\ Possible COVID-19 recurrence in an older patient: a case report
}

Antoine Garnier-Crussard ( $\boldsymbol{\nabla}$ antoine.garnier-crussard@chu-lyon.fr )

Hospices Civils de Lyon https://orcid.org/0000-0002-5611-3608

\section{Marine Haution}

Hospices Civils de Lyon

Mathilde Gueret-Du-Manoir

Hospices Civils de Lyon

Quitterie Reynaud

Hospices Civils de Lyon

Nathalie Freymond

Hospices Civils de Lyon

Maude Bouscambert-Duchamp

Hospices Civils de Lyon

Anne Conrad

Hospices Civils de Lyon

Claire Falandry

Hospices Civils de Lyon

\section{Case Report}

Keywords: COVID-19; recurrence; case report

Posted Date: June 15th, 2020

DOI: https://doi.org/10.21203/rs.3.rs-34694/v1

License: (c) (i) This work is licensed under a Creative Commons Attribution 4.0 International License.

Read Full License 


\section{Abstract}

Background: Novel coronavirus (COVID-19) pandemic cause by Severe Acute Respiratory Syndrome Coronavirus-2 (SARS-CoV-2) threatens the world for few months. Few cases of prolonged positivity of viral tests and clinical recurrence of COVID-19 have been described. We report the case of a 78-year-old woman with metastatic breast cancer who developed possible COVID-19 recurrence in a post-acute and rehabilitation unit.

Case presentation: A 78-year-old woman with metastatic breast cancer and hypertension developed COVID-19. After symptom improvement and RT-PCR negativation, she regained symptom (fever, fall) and lymphopenia on Day 26 and we note a turned positive RT-PCR even though she was tested positive for antibody against SARS-CoV-2. After the diagnosis of possible COVID-19 recurrence, she was transferred back to an acute "COVID-19" unit and she then quickly clinically recovered.

Conclusions: This clinical case allows us to discuss the risk of recurrence and possible specific causes in older patients. Moreover, prolonged symptoms and lymphopenia could be associated to worse outcomes in older patients. Finally, at a collective level, even if traces of virus detected by RT-PCR were not necessarily correlated with the contagiousness, the importance of possible COVID-19 recurrence in the care pathway for older adults must be taken into account, since they are often surrounded by frail older people.

\section{Background}

Novel coronavirus (COVID-19) pandemic caused by Severe Acute Respiratory Syndrome Coronavirus-2 (SARS-CoV-2) threatens the world for few months. Older patients with comorbidities are likely to be at increased risk of severe infections, cascade of complications, disability, and death [1, 2]. Their care courses must adapt permanently to the always renewed evidence on the pathogenesis of the disease. Very few cases of prolonged positivity of viral tests and clinical recurrence of COVID-19 have been described [3-8]. The case of a 78-year-old woman with COVID-19 possible recurrence in our post-acute and rehabilitation unit prompted us to discuss many issues that we decided to share, as geriatricians may have to face similar dilemmas.

\section{Case Presentation}

We report the case of a 78-year-old woman who had a history of breast carcinoma and bone metastases treated by letrozole (an aromatase inhibitor) and prednisolone (a corticosteroid) for few days, and hypertension, with long-term use of ramipril (an angiotensin-converting enzyme inhibitor).

After a 9-day history of fever and deteriorating general condition, she presented an acute respiratory distress syndrome, prompting her admission to the intensive care unit. Chest computed tomography 
revealed bilateral ground-glass lesions related to COVID-19, reaching $25 \%$ to $50 \%$ of the lungs (Figure) and nasopharyngeal swab detected SARS-CoV-2 with a quite high [33.2] cycle threshold using specific reverse transcription polymerase chain reaction (RT-PCR, cobas ${ }^{\circledR}$ SARS-CoV-2 Test, Roche Diagnostics, Switzerland). Clinical condition improved rapidly under oxygen therapy and increased corticotherapy. Fever and respiratory symptoms resolved on Day 19, a control nasopharyngeal swab was negative for SARS-CoV-2 detection on Day 23, allowing her to be transferred to a post-acute unit. Respiratory isolation measures were maintained. At Day 26 from the first symptoms, she presented fever relapse and fell. According to hemogram follow up, the lymphopenia that had reached nadir at $0.35 \otimes 10^{9} / \mathrm{L}$ at Day 18 and was improving $\left(0.60 \otimes 10^{9} / \mathrm{L}\right.$ at Day 25$)$ revealed a new decrease $\left(0.36 \otimes 10^{9} / \mathrm{L}\right)$. At the same time, a control chest computed tomography showed improvement in bilateral patchy ground glass opacities (Figure). Finally, RT-PCR performed on nasopharyngeal swab turned positive, with a quite high [32,7] cycle threshold, similar to the first RT-PCR. Notably, the patient was tested positive for IgG antibody against SARS-CoV-2 (SARS-CoV-2 IgG index 8.49, Architect, Abbott, USA).

No other respiratory viruses (Influenza A virus, Influenza B virus, Respiratory Syncytial virus, Picornavirus, Metapneumovirus, Parainfluenzae, Adenovirus) or bacteria (Mycoplasma pneumoniae, Chlamydia pneumoniae, Bordetella pertussis, Bordetella parapertussis) were detected by multiplex PCR (detected by FilmArray ${ }^{\circledR}$ Respiratory Panel 2 plus, Biomérieux, France). There was no sign of bacterial secondary infection or other source of infection. The patient showed no clinical signs of severity and was transferred back to an acute "COVID-19" unit. She then quickly clinically recovered.

\section{Discussion And Conclusions}

This example of COVID-19 possible recurrence appeals at facing major issues for older patients' care courses, at the individual and collective level.

In our view, the relapse of symptoms (fever), associated to lymphopenia and turned-positive RT-PCR in the present case, with no sign of other infection, could allow for the diagnosis of possible COVID-19 recurrence in this case.

At the individual level, this case alerts on the possibility of recurrence of symptoms and turned-positive RT-PCR in recovered patients, with positive serology. Some recent reports found positive RT-PCR tests in cases of recovered COVID-19 patients, regardless of symptoms [3-8]. Viral test positivity should be differentiated from clinical recurrence and from persistence of contagiousness. The pathophysiology is not clear and several mechanisms are hypothesized, such as technical issues, viral reactivation, lack of immunological control or reinfection $[4-6,9]$. Increasing age, male gender, hypertension and corticosteroid treatment, which we know are highly prevalent in older adults, were recently associated to delay in viral clearance [10]. The protective effect to viral infection in females is partly explained by estrogen and progesterone which could help to increase the innate and adaptive immune responses [10, 11]. This protection was probably impaired in this post-menopausal patient treated with anti-aromatase drug for breast cancer. Moreover, immune senescence, known to impair immune response to infectious 
diseases in older adults may participate to viral clearance delay [12]. A case of COVID-19 recurrence in a 48-year-old man was recently published [8]. As in the present case, the patient had anti-SARS-CoV-2 IgG, indicating that the acute phase of the disease and immunological reaction occurred, and suggesting that in some cases the presence of IgG antibodies could be not protective [8], although these suggestions should be taken with caution [9].

Another issue is that prolonged symptoms such as fever in older patients expose them to prolonged risk of complications, such as falls or delirium. Finally, our patient presented a persistent lymphopenia, with new decrease of lymphocytes count the day of the recurrence (at $0.36 \otimes 10^{9} / \mathrm{L}$ ). The correlation between lymphopenia and positive virus detection suggests the link between viral replication and viro-induced lymphocyte destruction. Proposed mechanisms leading to lymphopenia during COVID-19 are possibly multiple, including viral toxicity, inflammatory cytokines dysregulation, and metabolic disorders [13]. A prolonged lymphopenia, occurring in all lymphocyte subsets, including CD8+ and natural killer cells (antiviral function), and B cells (which differentiate into plasma cells able to produce neutralizing antibodies) [14], has been previously associated to higher mortality in older population and with an increased risk of secondary infections [15].

At the collective level, even if traces of virus detected by RT-PCR were not necessarily correlated with the contagiousness, this case raises the question of patients' care courses, since it implies the need for prolonged isolation, if reasonably practicable, until we have more data on such contagiousness. Geriatric patients with COVID-19 cumulate risk factors of prolonged viral shedding and of trans-contaminations at risk, since they are often surrounded by frail older people (older spouse, nursing home etc). There does not currently appear to have been any secondary contamination, but intra-hospital transmission of COVID-19 to frail older adults, in acute units, post-acute unit or long-term care would be dramatic.

There are some limitations in the present case report. The patient had only one negative RT-PCR before the relapse, while two consecutive negative RT-PCR [5] would have been better to talk about recovery. Moreover, the imputability of COVID-19 in the clinical presentation cannot be guaranteed, even if the association of fever, lymphopenia, turned-positive RT-PCR and the absence sign of other respiratory viruses or bacteria are highly suggestive of a possible COVID-19 recurrence.

In conclusion, geriatricians, who are already well aware of the seriousness of COVID-19, will also have to address the issue of possible recurrence, if it is confirmed. We need more studies, specifically in older patients, to define the risk factors for clinical or virologic recurrence, as well as the potential risk of contagiousness. It seems necessary in order to limit consequences of prolonged symptoms (such as fever) or lymphopenia, and to organize safely and effectively the care of older patients with COVID-19 across the whole geriatric pathway, from acute units to long-term care facilities and nursing homes. As the older patients are already the people most at risk of mortality and complications, the burden of the COVID-19 pandemic could be increasingly significant.

\section{Abbreviations}


COVID-19: coronavirus disease 2019

IgG: immunoglobulin G

RT-PCR: reverse transcription-polymerase chain reaction

SARS-CoV-2: severe acute respiratory syndrome coronavirus 2

\section{Declarations}

\section{Ethics approval and consent to participate}

Informed and written consent was obtained from the patient.

\section{Consent to publish}

Informed and written consent was obtained from the patient.

\section{Availability of data and materials}

$\mathrm{N} / \mathrm{A}$

\section{Competing interests}

None

\section{Funding}

None

\section{Authors' contributions}

- $A G C, M H, M G D M, Q R, M B D$ and $C F$ had a role in the acquisition of data and patient's monitoring

- $A G C$ and CF drafted the manuscript

- $\mathrm{MH}, \mathrm{MGDM}, \mathrm{QR}, \mathrm{NF}, \mathrm{MBD}, \mathrm{AC}, \mathrm{CF}$ revised the manuscript for intellectual content

- All authors have read and approved the manuscript

\section{Acknowledgements}

None

\section{References}

1. Garnier-Crussard A, Forestier E, Gilbert T, Krolak-Salmon P. Novel Coronavirus (COVID-19) Epidemic: What Are the Risks for Older Patients? J Am Geriatr Soc. 2020. doi:10.1111/jgs.16407. 
2. Shahid Z, Kalayanamitra R, McClafferty B, Kepko D, Ramgobin D, Patel R, et al. COVID-19 and Older Adults: What We Know. J Am Geriatr Soc. 2020. doi:10.1111/jgs.16472.

3. Yuan J, Kou S, Liang Y, Zeng J, Pan Y, Liu L. PCR Assays Turned Positive in 25 Discharged COVID-19 Patients. Clinical Infectious Diseases. 2020. doi:10.1093/cid/ciaa398.

4. Ye G, Pan Z, Pan Y, Deng Q, Chen L, Li J, et al. Clinical characteristics of severe acute respiratory syndrome coronavirus 2 reactivation. Journal of Infection. 2020;80:e14-7.

5. Xiao AT, Tong YX, Zhang S. False-negative of RT-PCR and prolonged nucleic acid conversion in COVID-19: Rather than recurrence. Journal of Medical Virology. doi:10.1002/jmv.25855.

6. Zhang J, Yan K, Ye H, Lin J, Zheng J, Cai T. SARS-CoV-2 turned positive in a discharged patient with COVID-19 arouses concern regarding the present standard for discharge. Int J Infect Dis. 2020. doi:10.1016/j.ijid.2020.03.007.

7. Yuan Y, Wang N, Ou X. Caution should be exercised for the detection of SARS-CoV-2, especially in the elderly. J Med Virol. 2020.

8. Loconsole D, Passerini F, Palmieri VO, Centrone F, Sallustio A, Pugliese S, et al. Recurrence of COVID19 after recovery: a case report from Italy. Infection. 2020. doi:10.1007/s15010-020-01444-1.

9. Kirkcaldy RD, King BA, Brooks JT. COVID-19 and Postinfection Immunity: Limited Evidence, Many Remaining Questions. JAMA. 2020. doi:10.1001/jama.2020.7869.

10. Chen X, Hu W, Ling J, Mo P, Zhang Y, Jiang Q, et al. Hypertension and Diabetes Delay the Viral Clearance in COVID-19 Patients. medRxiv. 2020;:2020.03.22.20040774.

11. Ghosh S, Klein RS. Sex drives dimorphic immune responses to viral infections. J Immunol. 2017;198:1782-90.

12. Nikolich-Zugich J, Knox KS, Rios CT, Natt B, Bhattacharya D, Fain MJ. SARS-CoV-2 and COVID-19 in older adults: what we may expect regarding pathogenesis, immune responses, and outcomes. GeroScience. 2020. doi:10.1007/s11357-020-00186-0.

13. Tan L, Wang Q, Zhang D, Ding J, Huang Q, Tang Y-Q, et al. Lymphopenia predicts disease severity of COVID-19: a descriptive and predictive study. Sig Transduct Target Ther. 2020;5:33.

14. Remy KE, Brakenridge SC, Francois B, Daix T, Deutschman CS, Monneret G, et al. Immunotherapies for COVID-19: lessons learned from sepsis. The Lancet Respiratory Medicine. 2020;:S2213260020302174.

15. Vulliamy PE, Perkins ZB, Brohi K, Manson J. Persistent lymphopenia is an independent predictor of mortality in critically ill emergency general surgical patients. Eur J Trauma Emerg Surg. 2016;42:755-60.

\section{Figures}




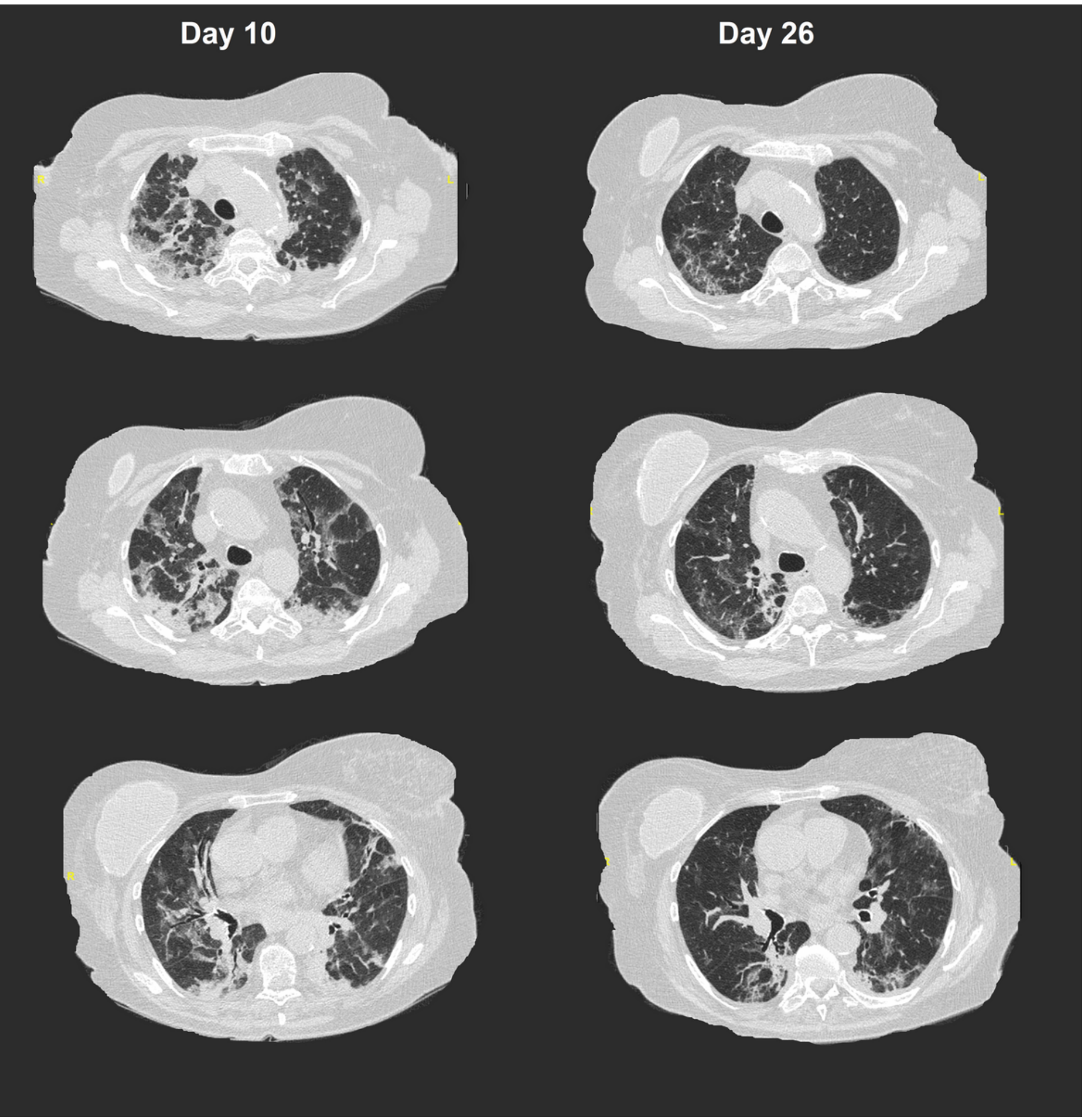

\section{Figure 1}

Chest computed tomography scans with bilateral patchy ground glass opacities related to COVID-19 showed a marked improvement between Day 10 and Day 26 from the first symptoms. 